\title{
p16 promoter hypermethylation is associated with increased risk of nasopharyngeal carcinoma
}

\author{
YANG SHAO $^{1}$, HONGGUO JIANG $^{2}$, XIAOMING WU $^{2}$, YING LUO $^{2}$ and WENRU TANG ${ }^{2}$ \\ ${ }^{1}$ Laboratory of Molecular Genetics of Aging and Tumor, Faculty of Life Science and Technology; \\ ${ }^{2}$ Laboratory of Molecular Genetics of Aging and Tumor, Faculty of Medicine, \\ Kunming University of Science and Technology, Kunming, Yunnan 650500, P.R. China
}

Received June 4, 2014; Accepted July 22, 2014

DOI: $10.3892 / \operatorname{mco} .2014 .396$

\begin{abstract}
The present study aimed to investigate the effects of p16 hypermethylation on the risk of nasopharyngeal carcinoma (NPC) quantitatively, through a meta-analysis of available case-control studies including malignant and normal NPC tissue samples. Crude odds ratios (ORs) with $95 \%$ confidence intervals (CIs) were extracted and pooled to assess the strength of the association between p16 hypermethylation and NPC risk. A total of 9 studies, including 406 NPC and 376 control cases, were identified for meta-analysis. Statistically significant ORs of p16 hypermethylation were obtained from the NPC and control groups $(\mathrm{OR}=19.53$; 95\% CI: 9.54-39.97; $\mathrm{P}=0.685)$. The meta-analysis indicated that p16 hypermethylation significantly increases NPC risk.
\end{abstract}

\section{Introduction}

Nasopharyngeal carcinoma (NPC) is a highly invasive and metastatic malignant tumor that originates from the epithelial cells lining the nasopharynx (1). Several histological entities exist, but undifferentiated NPC is the most frequent histological type. NPC differs significantly from other head and neck cancers due to its specific multifactorial etiology and geographical distribution. NPC is relatively rare in most parts of the world, particularly in Europe and North America; however, it has a high incidence in Southern China. Outside of these areas, intermediate incidences are observed in the Eskimo population of Arctic regions and in Arabians of North Africa, including Tunisia, Algeria and Morocco (2-4).

Recently, a number of epigenetic changes, such as methylation and histone deacetylation, have been found to be involved

Correspondence to: Dr Wenru Tang, Laboratory of Molecular Genetics of Aging and Tumor, Faculty of Medicine, Kunming University of Science and Technology, 727 Jing Ming Nan Road, Chenggong County, Kunming, Yunnan 650500, P.R. China

E-mail: twr@sina.com

Key words: p16 hypermethylation, nasopharyngeal cancer, meta-analysis in the tumorigenesis of NPC. In addition, growing evidence demonstrates that epigenetic changes contribute to this process by altering the functions of multiple genes playing critical roles in cell cycle regulation, apoptosis, signal transduction, adhesion and differentiation of the nasopharyngeal epithelial cells (5). The most prevalent epigenetic mechanism implicated in this type of cancer is the hypermethylation of $\mathrm{CpG}$ islands in the promoter regions of the genes.

p16, one of the most commonly inactivated tumor suppressor genes in human cancer, is a cyclin-dependent kinase $(\mathrm{CDK})$ inhibitor that regulates tumor cell progression through the G1 phase of the cell cycle (6). The downregulation of p16 expression due to promoter hypermethylation frequently occurs in NPC. Aberrant DNA hypermethylation has been recognized as a frequent molecular alteration in cancer $(7,8)$. This epigenetic modification occurs at the cytosines of $\mathrm{CpG}$ dinucleotide-rich regions (9), which are mostly unmethylated in normal tissues. The hypermethylation of $\mathrm{CpG}$ islands in the gene promoter regions of numerous tumor suppressor and DNA repair genes is associated with chromatin condensation, replication delay, inhibition of the initiation of transcription and gene silencing (10). Similar to a number of other genes, p16 is commonly inactivated by the hypermethylation of its CpG-rich promoter region (11). The association between p16 promoter methylation and NPC susceptibility has been extensively investigated; however, the results have been inconsistent. Thus, a comprehensive meta-analysis of the most recent and relevant studies was performed to identify statistical evidence of the association between p16 gene hypermethylation and NPC risk. Therefore, a systematic review and meta-analysis was conducted to evaluate this correlation quantitatively.

\section{Materials and methods}

Meta-analysis. This meta-analysis was performed according to the Preferred Reporting Items for Systematic Reviews and Meta-Analyses (12) and the recommendations of the Cochrane Collaboration (13). The study was approved by the Institutional Review Board of the Kunming University of Science and Technology.

Identification and eligibility of relevant studies. To identify eligible studies, the terms 'p16', 'methylation', 'nasopharyngeal 
Table I. Data from 9 studies on p16 methylation and nasopharyngeal cancer (NPC) risk included in this study.

\begin{tabular}{|c|c|c|c|c|c|c|}
\hline \multirow[b]{2}{*}{ First author } & \multirow[b]{2}{*}{ Year } & \multicolumn{2}{|c|}{ NPC cases $(n=406)$} & \multicolumn{2}{|c|}{ Controls (n=376) } & \multirow[b]{2}{*}{ (Refs.) } \\
\hline & & Methylated & Unmethylated & Methylated & Unmethylated & \\
\hline Tian et al & 2013 & 9 & 31 & 1 & 40 & (28) \\
\hline Challouf et al & 2012 & 12 & 24 & 4 & 158 & (29) \\
\hline Hutajulu et al & 2011 & 35 & 18 & 0 & 25 & $(30)$ \\
\hline Tan et al & 2013 & 6 & 13 & 0 & 3 & $(31)$ \\
\hline Xiang and Zhang & 2005 & 42 & 48 & 0 & 30 & (32) \\
\hline Wong et al & 2004 & 17 & 24 & 1 & 42 & (33) \\
\hline Chang et al & 2003 & 10 & 23 & 0 & 37 & (34) \\
\hline Tong et al & 2002 & 13 & 15 & 0 & 26 & (35) \\
\hline Kwong et al & 2002 & 17 & 16 & 0 & 6 & (36) \\
\hline Lo et al & 1996 & 6 & 27 & 0 & 3 & (37) \\
\hline
\end{tabular}

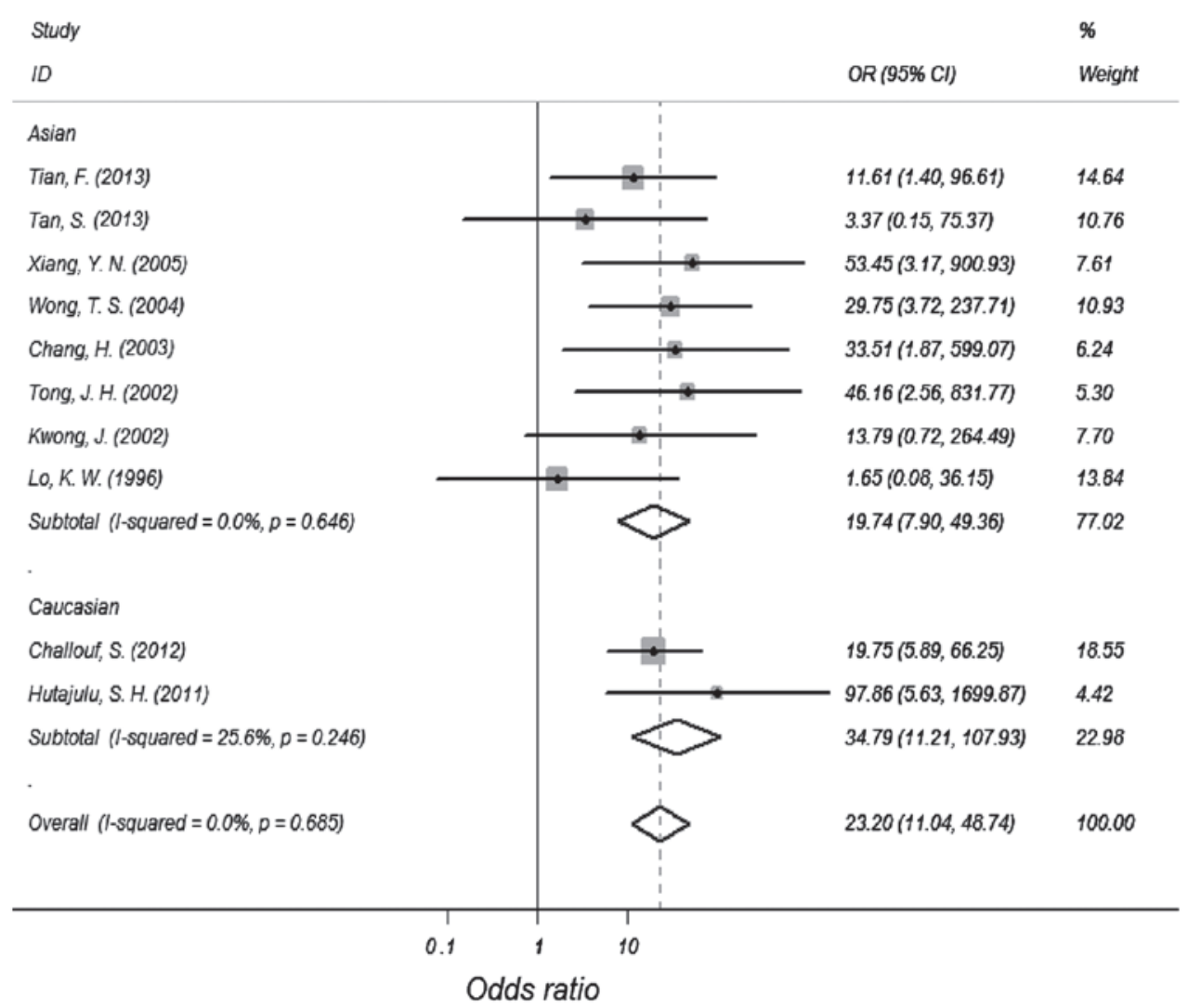

Figure 1. Forest plot of odds ratios (ORs) of nasopharyngeal cancer risk according to p16 promoter hypermethylation when compared to the control groups. The squares and horizontal lines correspond to each study-specific OR and 95\% confidence interval (CI). The area of the squares reflects the study-specific weight. The diamonds represent the pooled ORs and 95\% CIs.

cancer', 'nasopharyngeal tumor' and 'nasopharyngeal carcinoma' were searched for in PubMed, Web of Science and EBSCO with an English language restriction. The search results were updated until February 24, 2014. Only published full-text articles were included in the analysis. The following criteria were used in selecting eligible articles: i) independent case-control, malignant and benign nasopharyngeal tissue studies with reliable methods; ii) studies investigating p16 methylation and NPC risk; iii) sufficient data for estimating odds ratios (ORs) (14) with 95\% confidence intervals (CIs).
Data extraction. Shao and Tang independently conducted reviews by extracting data via a standardized approach. The publication information (year of publication and name of first author) and p16 hypermethylation rates were collected using standard data extraction forms. Any discrepancies were resolved through discussion.

Statistical analysis. The ORs and 95\% CIs were calculated to estimate the strength of the association between p16 hypermethylation and NPC risk. A Chi-square-based Q-test 
was performed to assess between-study heterogeneity. If $\mathrm{P}>0.10$, the studies were considered to lack heterogeneity and the pooled ORs were calculated using the fixed-effects model according to the Mantel-Haenszel method (15). Otherwise, the random-effect model was used according to the DerSimonian-Laird method (16). All the statistical analyses in the present study were performed using Review Manager 5 (http://tech.cochrane.org/revman) and Stata software ( version 8.2;StataCorp LP, College Station, TX, USA).

\section{Results}

Study characteristics. A total of 9 studies met the inclusion criteria and were included in the present meta-analysis. The studies involved 406 NPC and 376 control cases. When the same investigators reported results obtained from the same cohort of patients in several publications, only the largest series was included in the analysis. A cohort of patients was excluded due to duplicate reports. The main characteristics of the included studies are summarized in Table I.

p16 hypermethylation in NPC and control groups. Data regarding the comparison of p16 hypermethylation between the NPC and control groups were collected from 9 studies. The NPC and control groups included 167 (41.1\%) and $6(1.6 \%)$ p16 hypermethylated cases, respectively. The pooled analysis demonstrated that the ORs of the NPC group during p16 hypermethylation significantly increased compared to those of the controls (OR=19.53; 95\% CI: 9.54-39.97; $\mathrm{P}=0.685)$ (Fig. 1).

\section{Discussion}

The precise etiology of NPC (17) remains to be clearly elucidated; however, this disease has been found to be highly associated with specific geographical distributions, environmental factors and genetic alterations (18). $\mathrm{CpG}$ islands are located in the promoter or first exon region of genes and are normally unmethylated. Over the last few years, promoter hypermethylation has been recognised as a common mechanism underlying the inactivation of tumor suppressor genes in human cancer (19-21). However, in cancer cells, hypermethylation of these regions is associated with transcriptional silencing or a decrease in expression. The promoter hypermethylation of tumor suppressor genes, including p16, hMLH1 and VHL, has been established as a common mechanism for tumor suppressor gene inactivation in human cancer and has become a promising new molecular target for its detection. Aberrant DNA methylation is being increasingly recognized as a frequent molecular alteration in NPC. The product of the INK4A locus, p16, encodes a CDK (9) inhibitor that functions as a negative regulator of cyclin/CDK complexes. p16 binds preferentially to CDK4/6 and prevents the association of CDK4/6 with D-type cyclins, thus inhibiting $\mathrm{pRB}$ phosphorylation and progression through the cell cycle $(22,23)$. p16 also plays a significant role in the maintenance of normal cellular properties and prevention of centrosome dysfunction and genomic instability (24). p16 inactivation occurs during the early stage of carcinogenesis (25) and the loss of p16/RB activity occurs through various mechanisms, including the deletion, mutation and hypermethylation of p16 (23). Aberrant hypermethylation has been suggested to be a useful biomarker, with implications for NPC etiology, diagnosis and management. Gene-specific promoter alterations are common epigenetic aberrations in human NPC. However, the epigenetic changes in p16 gene hypermethylation specific to NPC etiology remain elusive. The present pooled analysis comprehensively assessed the correlation between p16 gene hypermethylation and the incidence of NPC based on 9 studies, which included 406 NPC cases and 376 controls. Using the pooled crude ORs from the studies, we demonstrated that p16 gene hypermethylation is associated with a 20.88 -fold increased risk of NPC compared to the control group.

The present study had several potential limitations. First, the possibility of information and selection bias and unidentified confounders cannot be completely excluded, as all the studies were observational. Second, the majority of the studies included in this meta-analysis were conducted in various ethnic groups.

In conclusion, despite the discrepancy in p16 hypermethylation in nasopharyngeal cancer and control groups, we observed that p16 hypermethylation is associated with an increased risk of NPC. p16 hypermethylation, which mediates the inactivation of the p16 gene, plays a significant role in nasopharyngeal carcinogenesis. Therefore, it may have useful clinical applications in the detection of NPC at the early stages. Currently available cancer therapies use DNA methylation inhibitors, including 5-aza-2'-deoxycytidine (26), to reactivate the repressed p16 gene. The reactivation of the p16 gene in cells with methylated genes restores normal cell growth control (27). According to the present study, the p16 promoter methylation status may be used for NPC diagnostic and therapeutic purposes.

\section{Acknowledgements}

This study was supported by the Planned Science and Technology Project of Yunnan Province (grant nos. 2012FB134 and 2011DH011).

\section{References}

1. Spano JP, Busson P, Atlan D, et al: Nasopharyngeal carcinomas: an update. Eur J Cancer 39: 2121-2135, 2003.

2. Trimeche M, Braham H, Ziadi S, Amara K, Hachana M and Korbi S: Investigation of allelic imbalances on chromosome $3 p$ in nasopharyngeal carcinoma in Tunisia: high frequency of microsatellite instability in patients with early-onset of the disease. Oral Oncol 44: 775-783, 2008.

3. Parkin DM, Bray F, Ferlay J and Pisani P: Estimating the world cancer burden: Globocan 2000. Int J Cancer 94: 153-156, 2001.

4. Razak AR, Siu LL, Liu FF, Ito E, O'Sullivan B and Chan K: Nasopharyngeal carcinoma: the next challenges. Eur J Cancer 46: 1967-1978, 2010.

5. Yoshizaki T: Promotion of metastasis in nasopharyngeal carcinoma by Epstein-Barr virus latent membrane protein-1. Histol Histopathol 17: 845-850, 2002.

6. Li S, Rong M and Iacopetta B: DNA hypermethylation in breast cancer and its association with clinicopathological features. Cancer Lett 237: 272-280, 2006.

7. Wajed SA, Laird PW and DeMeester TR: DNA methylation: an alternative pathway to cancer. Ann Surg 234: 10-20, 2001.

8. Baylin SB and Herman JG: DNA hypermethylation in tumorigenesis: epigenetics joins genetics. Trends Genet 16: 168-174, 2000.

9. Zhou J, Cao J, Lu Z, Liu H and Deng D: A 115-bp MethyLight assay for detection of p16 (CDKN2A) methylation as a diagnostic biomarker in human tissues. BMC Med Genet 12: 67, 2011. 
10. Esteller M, Corn PG, Baylin SB and Herman JG: A gene hypermethylation profile of human cancer. Cancer Res 61: 3225-3229, 2001.

11. Di Vinci A, Perdelli L, Banelli B, et al: p16 ${ }^{\text {INK4a }}$ promoter methylation and protein expression in breast fibroadenoma and carcinoma. Int J Cancer 114: 414-421, 2005.

12. Moher D, Liberati A, Tetzlaff J, Altman DG and PRISMA Group: Preferred reporting items for systematic reviews and meta-analyses: the PRISMA statement. Ann Intern Med 151: 264-269, 2009.

13. Chalmers I: The Cochrane collaboration: preparing, maintaining, and disseminating systematic reviews of the effects of health care. Ann NY Acad Sci 703: 156-165, 1993.

14. Lee S, Kim WH, Jung HY, Yang MH and Kang GH: Aberrant $\mathrm{CpG}$ island methylation of multiple genes in intrahepatic cholangiocarcinoma. Am J Pathol 161: 1015-1022, 2002.

15. Jose S, George PS and Mathew A: Assessment of confounding and interaction using the mantel-haenszel risk estimation method.Asian Pac J Cancer Prev 9: 323-325, 2008.

16. Böhning D, Malzahn U, Dietz E, et al: Some general points in estimating heterogeneity variance with the DerSimonian-Laird estimator. Biostat 3: 445-457, 2002.

17. Ayadi W, Karray-Hakim H, Khabir A, et al: Aberrant methylation of p16, DLEC1, BLU and E-cadherin gene promoters in nasopharyngeal carcinoma biopsies from Tunisian patients. Anticancer Res 28: 2161-2167, 2008.

18. Ho JH: Stage classification of nasopharyngeal carcinoma: a review. IARC Sci Publ 20: 99-113, 1978.

19. Rountree MR, Bachman KE, Herman JG and Baylin SB: DNA methylation, chromatin inheritance, and cancer. Oncogene 20 : 3156-3165, 2001.

20. Widschwendter M and Jones PA: The potential prognostic, predictive, and therapeutic values of DNA methylation in cancer. Clin Cancer Res 8: 17-21, 2002.

21. Zou HZ, Yu BM, Wang ZW, et al: Detection of aberrant p16 methylation in the serum of colorectal cancer patients. Clin Cancer Res 8: 188-191, 2002.

22. Rocco JW and Sidransky D: p16 (MTS-1/CDKN2/INK4a) in cancer progression. Exp Cell Res 264: 42-55, 2001.

23. Sherr CJ and McCormick F: The RB and $\mathrm{p} 53$ pathways in cancer. Cancer Cell 2: 103-112, 2002.

24. McDermott KM, Zhang J, Holst CR, Kozakiewicz BK, Singla V and Tlsty TD: p16 $6^{\mathrm{INK} 4 \mathrm{a}}$ prevents centrosome dysfunction and genomic instability in primary cells. PLoS Biol 4: e51, 2006.

25. Zou XP, Zhang B, Zhang XQ, Chen M, Cao J and Liu WJ Promoter hypermethylation of multiple genes in early gastric adenocarcinoma and precancerous lesions. Hum Pathol 40 $1534-1542,2009$.
26. Hassler MR, Klisaroska A, Kollmann K, et al: Antineoplastic activity of the DNA methyltransferase inhibitor 5-aza-2'-deoxycytidine in anaplastic large cell lymphoma. Biochimie 94: 2297-2307, 2012

27. Bender CM, Pao MM and Jones PA: Inhibition of DNA methylation by 5-aza-2'-deoxycytidine suppresses the growth of human tumor cell lines. Cancer Res 58: 95-101, 1998.

28. Tian F, Yip SP, Kwong DL, Lin Z, Yang Z and Wu VW: Promoter hypermethylation of tumor suppressor genes in serum as potential biomarker for the diagnosis of nasopharyngeal carcinoma. Cancer Epidemiol 37: 708-713, 2013.

29. Challouf S, Ziadi S, Zaghdoudi R, Ksiaa F, Ben Gacem R and Trimeche M: Patterns of aberrant DNA hypermethylation in nasopharyngeal carcinoma in Tunisian patients. Clin Chim Acta 413: 795-802, 2012.

30. Hutajulu SH, Indrasari SR, Indrawati LP, et al: Epigenetic markers for early detection of nasopharyngeal carcinoma in a high risk population. Mol Cancer 10: 48, 2011.

31. Tan S, Sun C, Wei X, et al: Quantitative assessment of lung cancer associated with genes methylation in the peripheral blood. Exp Lung Res 39: 182-190, 2013.

32. Xiang YN and Zhang WY: The clinical significance of p16 protein non-expression and p16 gene inactivation by deletions and hypermethylation in nasopharyngeal carcinoma. Chin J Pathol 34: 358-361, 2005 (In Chinese).

33. Wong TS, Kwong DL, Sham JS, Wei WI, Kwong YL and Yuen AP: Quantitative plasma hypermethylated DNA markers of undifferentiated nasopharyngeal carcinoma. Clin Cancer Res 10: 2401-2406, 2004.

34. Chang H, Yi B, Li L, et al: Methylation of tumor associated genes in tissue and plasma samples from liver disease patients. Exp Mol Pathol 85: 96-100, 2008

35. Tong JH, Tsang RK, Lo KW, et al: Quantitative Epstein-Barr virus DNA analysis and detection of gene promoter hypermethylation in nasopharyngeal (NP) brushing samples from patients with NP carcinoma. Clin Cancer Res 8: 2612-2619, 2002.

36. Kwong J, Lo KW, To KF, Teo PM, Johnson PJ and Huang DP: Promoter hypermethylation of multiple genes in nasopharyngeal carcinoma. Clin Cancer Res 8: 131-137, 2002.

37. Lo KW, Cheung ST, Leung SF, et al: Hypermethylation of the p16 gene in nasopharyngeal carcinoma. Cancer Res 56: 2721-2725, 1996. 